\title{
Tissue classification in microscope imaging based on a discrete-band spectroscopy
}

\author{
Gilwon Yoon $^{\mathrm{a}, *}$ and Hyejeong Kim ${ }^{\mathrm{b}}$ \\ ${ }^{a}$ Department of Electronic and Information, Seoul National University of Science and Technology, \\ Seoul, Korea \\ ${ }^{\mathrm{b}}$ Institute for Biomedical Electronics, Seoul National University of Science and Technology, Seoul, \\ Korea
}

\begin{abstract}
Automatic classification of tissue types has a potential use in endoscopic or microscopic imaging. For this study, the microscope images from composite tissue samples were measured. Different shades of red meat such as beef and pork and bloodless tissues such as chicken breast and fat were chosen to provide with similar and contrasted colors. We applied a partial least squares discriminant analysis (PLS-DA) to classify the tissue type of the image pixels. With the RGB color images that are usually available in the hospital, we could classify beef, pork and chicken with an accuracy of only $85.7 \%$ and $80 \%, 60 \%$ and $11.4 \%$, respectively. There was an apparent limitation in differentiating the tissue type due to the spectral overlapping of RGB colors. To increase the classification accuracy, band-pass filtered images were taken at the center frequencies of 414, 542, 655 and $832 \mathrm{~nm}$ without any spectral overlapping. Using these discrete-band spectral images, the classification accuracy reached to all $100 \%$ except for beef that was $96 \%$. In capsule endoscopy where the amount of image data is prohibitively large, automatic detection of bleeding or cancerous region is of great interest and we believe that this method can be applicable in real time monitoring.
\end{abstract}

Keywords: Classification, partial least squares, spectroscopy, medical image, endoscopy

\section{Introduction}

Automatic detection of a particular tissue type in medical imaging can be of great use especially for capsule endoscopy. Once a patient swallows an endoscopic capsule, it provides image in the gastrointestinal track and generates a huge amount of image data to be processed. For an example of MiroCam ${ }^{\mathrm{TM}}$, 118,800 color pictures with $320 \times 320$ pixels are recorded during 11-hour recording [6]. This particular system has three frames per second. It is not probable for a physician to attend during the whole time of monitoring. Often to find bleeding spot or cancerous region is of primary interest. When the physician has to diagnose the pictures, probably one should exert his or her substantial amount of time and concentrated efforts.

There have been investigations on tissue diagnosis in the field of medical imaging $[1,11,14,16]$. Recently there have been two noteworthy approaches. One is so-called the narrow band imaging (NBI). Instead of viewing the endoscope image as it is, the image is filtered through narrow-band optical filters. Microvascular structures were enhanced when 415 and $540 \mathrm{~nm}$ with the bandwidths between 20

\footnotetext{
${ }^{*}$ Corresponding author: Gilwon Yoon, Department of Electronic and Information, Seoul National University of Science and Technology, 138 Gongneung gil, Nowon-gu, Seoul 139-743, Korea. Tel.: +82 2970 6419; Fax: +82 2979 7903; E-mail: gyoon@seoultech.ac.kr.
} 
and $30 \mathrm{~nm}$ were applied [5]. Different wavelength bands were also used depending on clinical purposes $[4,9,20]$. All are in the same fashion which is looking through the optical filters. The other approach, called hyperspectral imaging, was originated from remote sensing [13]. Classification and target detection can be made from the satellite images having spectral information of many very narrow discrete wavelengths. Hyperspectral imaging has extended its applications into microscope image and poultry skin tumor detection $[3,7]$. Hyperspectral imaging has remained in the medical research area while NBI has been practiced in clinics.

Visible and infrared spectroscopy has been applied in cancer detection, tissue differentiation and the prediction of target concentration, etc. [10,15,17]. In this study, we are going to combine NBI with spectroscopy. NBI is subject to physician's diagnosis who examines the narrow-band images. Multivariate analysis can be a powerful tool for classification and target detection. It is our concept to perform spectroscopic analysis on each pixel in the medical image. Normally, a color CCD or CMOS camera which is used as the image detector in the endoscopy provides with three color information. They are red (R), green (G) and blue (B). R, G and B at each pixel can be regarded as three spectral band measurements. As another experiment, we measured the filtered images at several discrete frequencies. Then we applied a method known as partial least squares (PLS)-discriminant analysis (DA). PLS-DA has been used in classification such as endometrium sections based on near infrared spectra and pathology classification of Raman maps [8,19].

\section{Classification}

We applied a statistical analysis method to classify whether a pixel in the image belong to a particular type of tissue. Multivariate analysis of principal component regression (PCR) and partial least squares regression (PLSR) is the basis of PLS-DA. For image measurement, we used the samples that were made using four different tissues. They are beef (B), pork (P), chicken breast (C) and fat (F). Figure 1 shows a microscope image taken by a color CCD camera. B, P, C and F are the tissue types that we want to classify.

$$
\begin{aligned}
\left(\begin{array}{cccc}
y_{11} & y_{12} & \cdots & y_{1 q} \\
y_{21} & y_{22} & \cdots & y_{2 q} \\
\vdots & \vdots & \ddots & \vdots \\
y_{m 1} & y_{m 2} & \cdots & y_{m q}
\end{array}\right)= & \left(\begin{array}{cccc}
x_{11} & x_{12} & \cdots & x_{1 n} \\
x_{21} & x_{22} & \cdots & x_{2 n} \\
\vdots & \vdots & \ddots & \vdots \\
x_{m 1} & x_{m 2} & \cdots & x_{m n}
\end{array}\right)\left(\begin{array}{cccc}
b_{11} & b_{12} & \cdots & b_{1 q} \\
b_{21} & b_{22} & \cdots & b_{2 q} \\
\vdots & \vdots & \ddots & \vdots \\
b_{n 1} & b_{n 2} & \cdots & b_{n q}
\end{array}\right) \\
& +\mathbf{e} \text { (matrix). }
\end{aligned}
$$

The matrix y represents tissue classes or categories. $q$ is the number of the tissue classes. In our case, $q$ is four where the subscript 1 of $y_{i 1}$ represents beef, 2 for pork $\left(y_{i 2}\right), 3$ for chicken breast $\left(y_{i 3}\right)$ and 4 for fat $\left(y_{i 4}\right)$. The subscript, $i(i=1, \ldots, m)$, represents the $i$ th pixel. $m$ is the number of pixels. The pixels are two-dimensional in the image, but equation (1) shows the pixels in one-dimension from 1 to $m$ for a simpler mathematical expression. The matrix, $\mathbf{x}$ represents the spectral intensity where $n$ is the number of spectral bands. Therefore, $n$ is 3 for the RGB color image where the subscript 1 of $x_{i 1}$ represents R, 2 for $\mathrm{G}\left(x_{i 2}\right)$ and 3 for B $\left(x_{i 3}\right)$. For example, $x_{i 2}$ denotes the intensity of $\mathrm{G}$ for the $i$ th pixel. In the case of the filtered images using optical filters instead of RBG color, $x_{i j}$ will represent the $j$ th filtered spectral intensity for the $i$ th pixel. $\mathbf{b}$ is the coefficient matrix and $\mathbf{e}$ is the residuals. The key point is how to find b which minimizes e. 


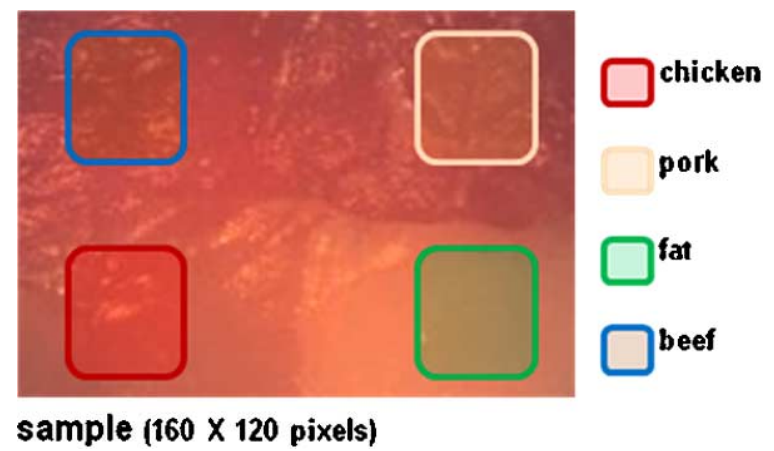

Fig. 1. A microscope image of a composite tissue sample measured by a RGB color CMOS camera. (Colors are visible in the online version of the article; http://dx.doi.org/10.3233/SPE-2011-0528.)

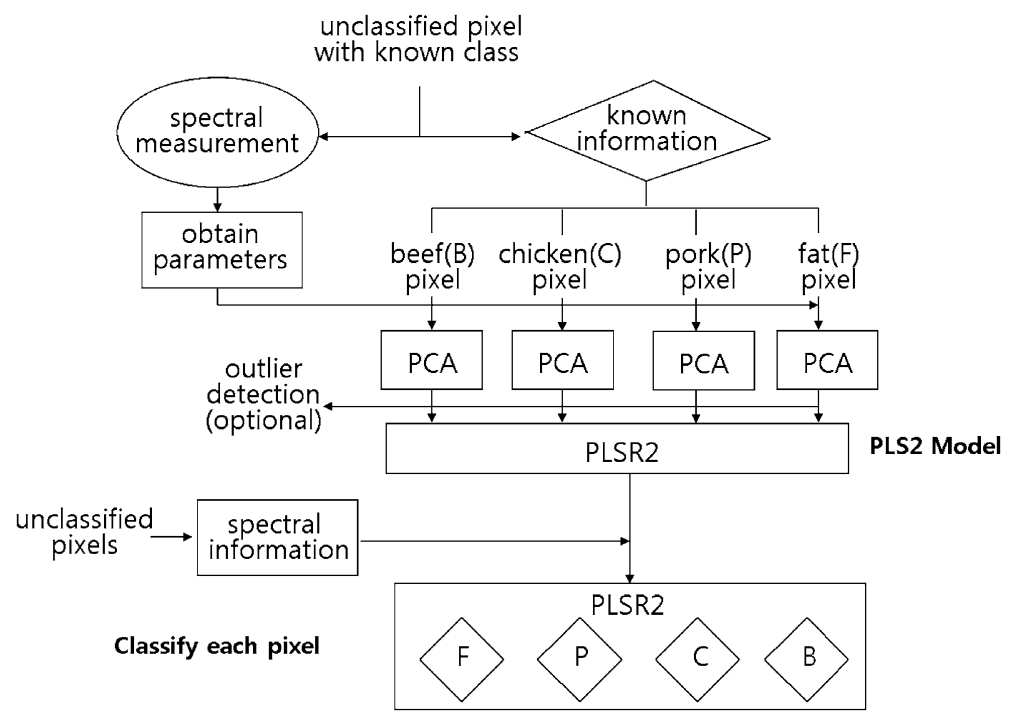

Fig. 2. A flow chart of classification based on partial least squares discriminant analysis (PLS-DA).

PCR decomposes the matrix $\mathbf{x}$ based on principal component analysis (PCA). The advantage of PCAdecomposition is to obtain a non-singular inverse matrix all the time unlike multiple linear regressions (MLR) and to provide a solution with no fail. However, one has to pay attention to the fact that the matrix is expressed by a limited number of principal components. PLSR decomposes $\mathbf{y}$ and $\mathbf{x}$ simultaneously using PCA. $\mathbf{x}$ is decomposed in some relevance with $\mathbf{y}$ unlike PCR. PLSR is known to be more robust and to have better predictive power compared with PCR $[2,12]$.

Figure 2 summarizes these processes. $\mathbf{b}$ is computed through PLS2 modeling. PLS regression fits to one variable. In our study, the regression is made to four variables (B, P, C and F) unlike PLS. We used the commercial software called The Unscrambler 9.7 (CAMO Software Inc., Norway). PLS-DA adapts a binary discriminant variable $\left(Y_{\mathrm{d}}\right)$ for $\mathbf{y}$. In this case, the discriminant variable, $Y_{\mathrm{d}}$, is assigned as either 1 or 0 (Yes $=1$, No $=0$ ) as reference value in $\mathbf{y}$. Binary encoding was used for the class descriptors. For the example of a beef pixel, beef variable is assigned as 1 and the rest variables of chicken, pork and fat equal to 0. PLS2 model with the known class information at each pixel is built. Through this process, the B, P, C and F models are constructed simultaneously. Then we apply the models to the pixels and 
checked to which class the pixel belonged. $Y_{\mathrm{d}} \geqslant 0.5$ is considered to be a class member and $Y_{\mathrm{d}}<0.5$ is not the member. We checked all four classes using the PLS2 model for each pixel. As example, we obtain $Y_{\mathrm{d}}$ and its standard deviation (SD) once beef model is run for a pixel. When $Y_{\mathrm{d}}$ is bigger than 0.5 , the pixel is considered to be beef. The rest models of chicken, pork and fat should be also run for the same pixel. This process is illustrated in Fig. 2.

However, there are varying degrees of certainty level (CL). For example, they are of the same class whether $Y_{\mathrm{d}}$ is 1.0 or 0.6 . Even for the same value of $Y_{\mathrm{d}}$, SD value is not the same. The lower end value is less than 0.5 when $Y_{\mathrm{d}}$ is 0.6 and the SD is 0.2 . Recall that $Y_{\mathrm{d}}$ is $0.6 \pm 0.2$. We introduced three certainty levels; CL1 as 'satisfactory' if the lower end value of $Y_{\mathrm{d}}$ is bigger than 0.5 , CL2 as 'marginally satisfactory' if it is between 0.5 and 0.25 , CL3 as 'poorly satisfactory' if lower than 0.25 . Regardless of CLs, class determination is the same as long as $Y_{\mathrm{d}}$ value is higher than 0.5 . Finally, each pixel can be diagnosed as one of the followings; $\mathrm{B}, \mathrm{C}, \mathrm{P}, \mathrm{F}, \mathrm{B} / \mathrm{C}, \mathrm{B} / \mathrm{P}, \mathrm{B} / \mathrm{F}, \mathrm{C} / \mathrm{P}, \mathrm{C} / \mathrm{F}, \mathrm{P} / \mathrm{F}, \mathrm{B} / \mathrm{C} / \mathrm{P}, \mathrm{B} / \mathrm{C} / \mathrm{F}, \mathrm{B} / \mathrm{P} / \mathrm{F}, \mathrm{C} / \mathrm{P} / \mathrm{F}$, $\mathrm{B} / \mathrm{C} / \mathrm{P} / \mathrm{F}$, none of these. Notation goes as follows. $\mathrm{C}$ is classified as chicken. $\mathrm{B} / \mathrm{C}$ is classified as beef as well as chicken. $\mathrm{B} / \mathrm{C} / \mathrm{F}$ is diagnosed as beef, chicken and fat at the same time. None of these is the case where there is no evidence of belonging to any class.

\section{Experimental section}

We did two different experiments. One was with RGB color images. The advantage of using the RGB color is that many endoscopic or microscopic images are based on RGB color. RGB color is readily available without further instrumentation or modification to the existing system. We wanted to investigate how well RGB color performs in tissue classification. In the second experiment, filtered images are measured. We inserted several filters in front of a monochrome CMOS camera. In this way, we took the bandpass-filtered images.

A size of $10 \times 10 \mathrm{~mm}$ composite tissue was prepared as sample. Fresh tissues of $2 \mathrm{~mm}$ thickness were obtained at the butcher's. Beef was sirloin, chicken was of breast part and the belly portion of pork was cutout. Fat portion of pork belly was taken out to be used as fat. Immediately after bringing them to the laboratory, each slice was cut to a size of $5 \times 5 \mathrm{~mm}$. They were placed together on the microscope slide as shown in Fig. 1. A cover slice was place on the top of sample. A microscope (SMZ-168, Motic ${ }^{\mathrm{TM}}$ ) and color CMOS camera (Moticam-1000 ${ }^{\mathrm{TM}}$ ) were set at a magnification of 10. Figure 1 shows the picture of the sample. The camera resolution was $160 \times 120(19,200$ pixels $) .10 \times 10$ detector pixels were put together as one unit area and there were a total of 192 units. Classification was made for each unit instead of one pixel. A software (Matlab ${ }^{\mathrm{TM}}$, The Mathworks Inc., USA) was used to get R, G and B colors whose values were retrieved based on the CIE XYZ color matching functions [18]. 35 units per tissue which made a total of 140 units were analyzed.

As for bandpass-filtered images, the center frequencies were 414, 542, 655 and $832 \mathrm{~nm}$ and their full bandwidths were 46, 27, 15 and $37 \mathrm{~nm}$, respectively. Each filter (single bandpass filter, Semrock Inc.) had a spectral profile that was very flat and the roll-off was very rapid. Unlike RGB colors, there was no spectral overlapping. Figure 3 shows the spectral profiles. Their spectral ranges covered the almost entire CMOS detector range (Infinity ${ }^{\mathrm{TM}} 1-1$ ). Figure 4 shows the microscope tissue images pictured through the filters. The detector resolution was $120 \times 100$ and we arranged 100 units by grouping the detector elements (Fig. 5).

Intensity at the near infrared of $832 \mathrm{~nm}$ is very weak due to two reasons. First, the CMOS quantum efficiency in the near infrared is very low. Secondly, the microscope illumination was mostly on the 
$414 \mathrm{~nm}$ with $46 \mathrm{~nm}$ bandwidth

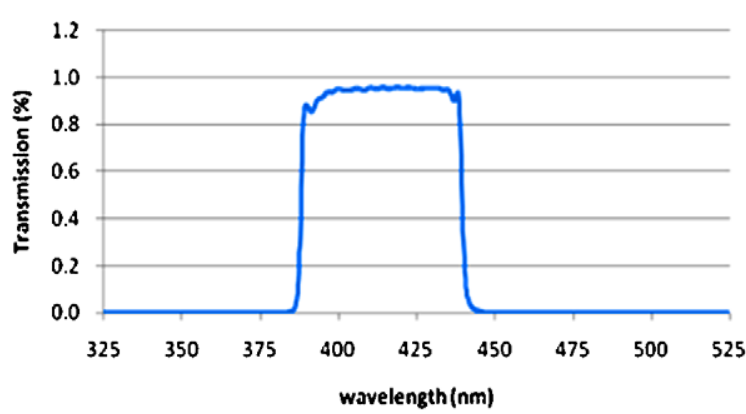

$655 \mathrm{~nm}$ with $15 \mathrm{~nm}$ bandwidth

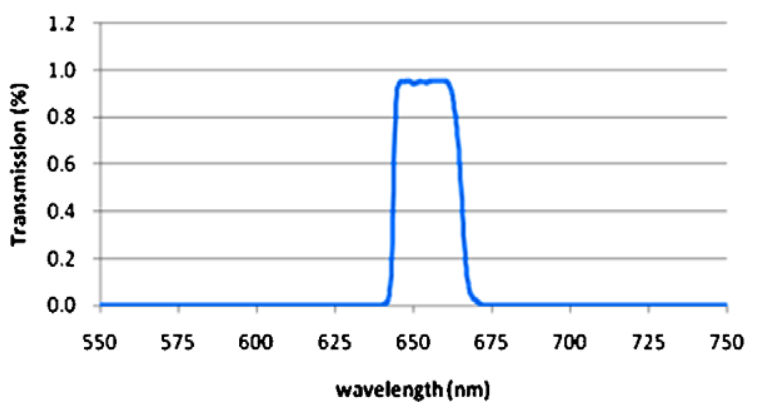

$542 \mathrm{~nm}$ with $27 \mathrm{~nm}$ bandwidth

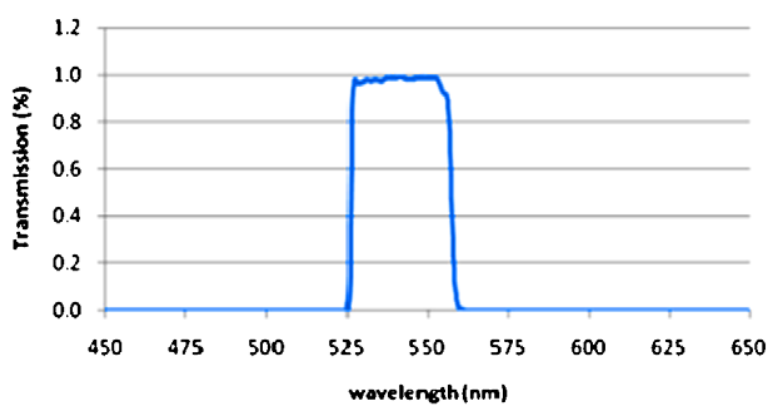

$832 \mathrm{~nm}$ with $37 \mathrm{~nm}$ bandwidth

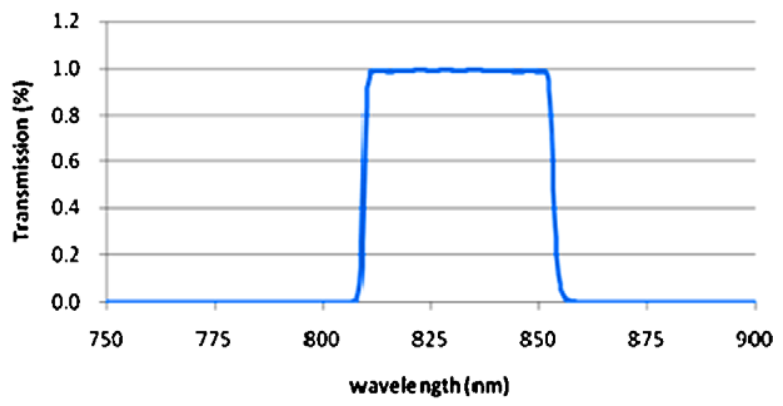

Fig. 3. Characteristics of filter frequency and transmission (single bandpass filter, Semrock Inc.). These filters were used to take filtered images of the microscope using a monochrome CMOS camera. (Colors are visible in the online version of the article; http://dx.doi.org/10.3233/SPE-2011-0528.)
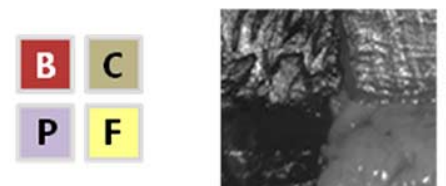

$414 \mathrm{~nm}$

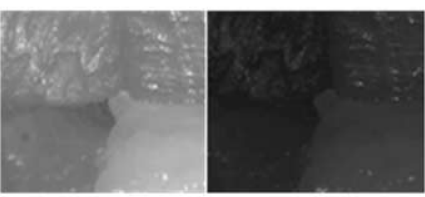

$655 \mathrm{~nm}$

832nm
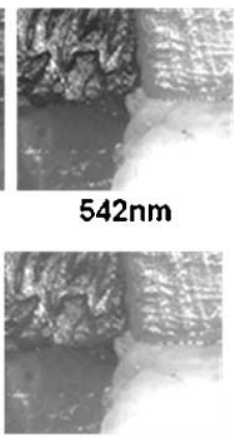

No filter

Fig. 4. Microscope pictures obtained from different filters where $\mathrm{B}$ indicates beef, $\mathrm{C}$ - chicken breast, $\mathrm{P}-$ pork and $\mathrm{F}-$ fat. (Colors are visible in the online version of the article; http://dx.doi.org/10.3233/SPE-2011-0528.)

visible spectrum. We had to install an extra halogen lamp to reinforce near infrared light and set much longer exposure times. The image shown in Fig. 4 had different exposure times. $832 \mathrm{~nm}$ image was still dark compared to other wavelengths'. Before PLS-DA, we had two different data preprocessing. One was the compensation of the quantum efficiency, filter transmission and exposure time. The other one was the normalization by the largest value of each wavelength. However, two different data preprocessing gave almost identical results. 

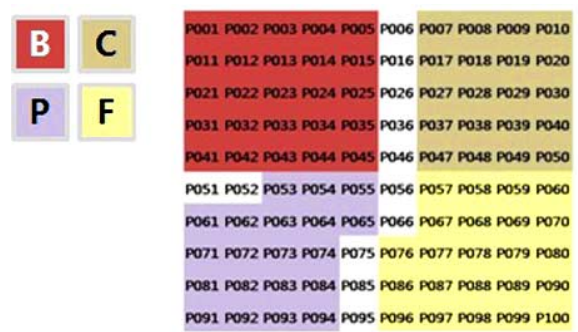

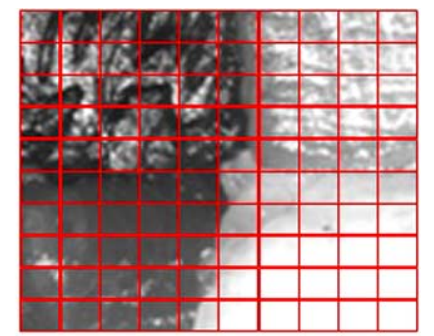

Fig. 5. The microscope image was assigned by units. Some units have more than one tissue type called mixed type. (Colors are visible in the online version of the article; http://dx.doi.org/10.3233/SPE-2011-0528.)

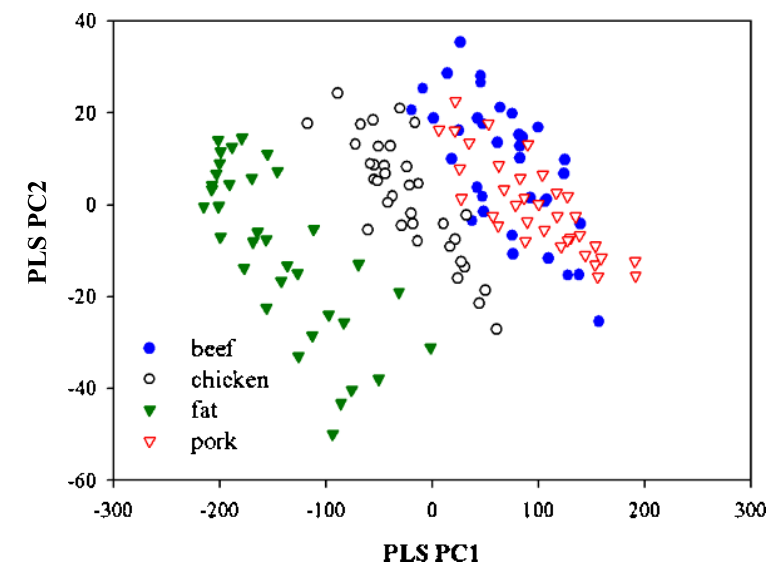

Fig. 6. PLS score plot using RGB color information. Each point in the figure corresponds to each unit. (Colors are visible in the online version of the article; http://dx.doi.org/10.3233/SPE-2011-0528.)

\section{Results and discussion}

\subsection{Classification using RGB colors}

PCA for each tissue showed that fat was separated from the rest and that others were somewhat overlapping in the PCA score plot. It indicates that fat was easier to be classified. We did not remove any outliers during PCA and PLS analysis. In general, the solutions were converged mostly within four principal components, sometimes up to five. However, there was not much contribution in computation from higher than three principal components. Figure 6 shows an example of PLS score plots with principal component (PC). Fat units are distributed towards the left and slightly lower corner. They are somewhat apart from the rest. Beef, pork and chicken are rather sharing the space. From the PLS score plot, it appeared that classification may be not very accurate though fat had a better chance of identification.

The results are summarized in Table 1. Fat was classified with the highest accuracy, $85.7 \%$. Also Certainty Level (CL) was very high. The rest of fat pixels, $14.3 \%$, were falsely identified as chicken. In the score plot, chicken were closer to fat and both had almost no blood (no red color). It was very difficult to classify chicken. Only $11.4 \%$ of chicken were identified and $82.9 \%$ belonged to none of four tissues. All 11.4\% classification was poorly satisfactory (CL3). It appears that chicken breast did not have a distinctive spectral feature. Beef was classified with an accuracy of $80 \%$ and the rest of 
Table 1

Classification accuracy of the tissue type based on RGB color information

\begin{tabular}{|c|c|c|c|c|c|}
\hline & & Correctl & edicted class & & \\
\hline & Beef & Chicken & Fat & Pork & None \\
\hline$\overline{\text { Beef }}$ & $\begin{array}{c}80.0 \\
(\mathrm{CL} 1=31.4, \\
\text { CL2 }=42.9, \\
\text { CL3 }=5.7)\end{array}$ & 0.0 & 0.0 & 20.0 & 0.0 \\
\hline Chicken & 2.9 & $\begin{array}{c}11.4 \\
(\mathrm{CL} 1=0, \\
\mathrm{CL} 2=0, \\
\text { CL3 = 11.4) }\end{array}$ & 2.9 & 0.0 & 82.9 \\
\hline Fat & 0.0 & 14.3 & $\begin{array}{c}85.7 \\
(C L 1=80, \\
\text { CL2 }=5.7, \\
\text { CL3 }=0)\end{array}$ & 0.0 & 0.0 \\
\hline Pork & 14.3 & 0.0 & 0.0 & $\begin{array}{c}60.0 \\
(\mathrm{CL} 1=5.7, \\
\text { CL2 }=42.9, \\
\text { CL3 }=11.4)\end{array}$ & 25.7 \\
\hline
\end{tabular}

Notes: $\mathrm{B}$ - beef, $\mathrm{C}$ - chicken breast, $\mathrm{P}$ - pork, $\mathrm{F}$ - fat and none - no class is identified. CL certainty level, CL1 - satisfactory, CL2 - marginally satisfactory, CL3 - poorly satisfactory.

beef were regarded as pork. Color-wise beef and pork are alike as they contain blood while beef was redder. That may be why beef was better classified. Overall, only with RGB color, classification was not very successful. Poor classification appears to come from the fact that RGB colors are overlapping substantially in the frequency domain.

\subsection{Classification using discrete-filtered images}

Figure 7 shows the PC plot based on PLS. The distribution of each tissue type was given in terms of PC1 and PC2. In the score plot, all four tissues are apart from any other tissues. It means that PLS models are separated and that possibly higher classification accuracies may be achieved. Results are given in Table 2. Classification accuracy was 96, 100, 100 and $100 \%$ for beef, chicken, pork and fat, respectively. Almost all classifications were in satisfactory level (CL1). Four percents of beef were classified as both beef and pork. When five spectral regions including the no-filtered image were used, the classification accuracy was rather decreased to $92,100,95$ and $100 \%$, respectively. Spectral redundancy might have caused a collinearity problem although additional spectrum was added to PLS modeling.

There were some units that lied in the boundaries. We call these as 'mixed' units. As shown in the PLS score plot (Fig. 7), the mixed units shared the space interfering with other types of tissue. Four units between beef and chicken were classified as beef. One unit (P046) which had three tissues of beef, chicken and fat was identified as beef. We need to comment that P046 had a smaller portion of chicken. P051 and P052 were between beef and pork. But their portions of pork were higher (approximately over $70 \%$ ) and P051 and P052 were classified as pork. Two units (P056 and P066) between pork and fat with more fat portions were classified as none. P75 and P85 were between pork and fat with more pork 


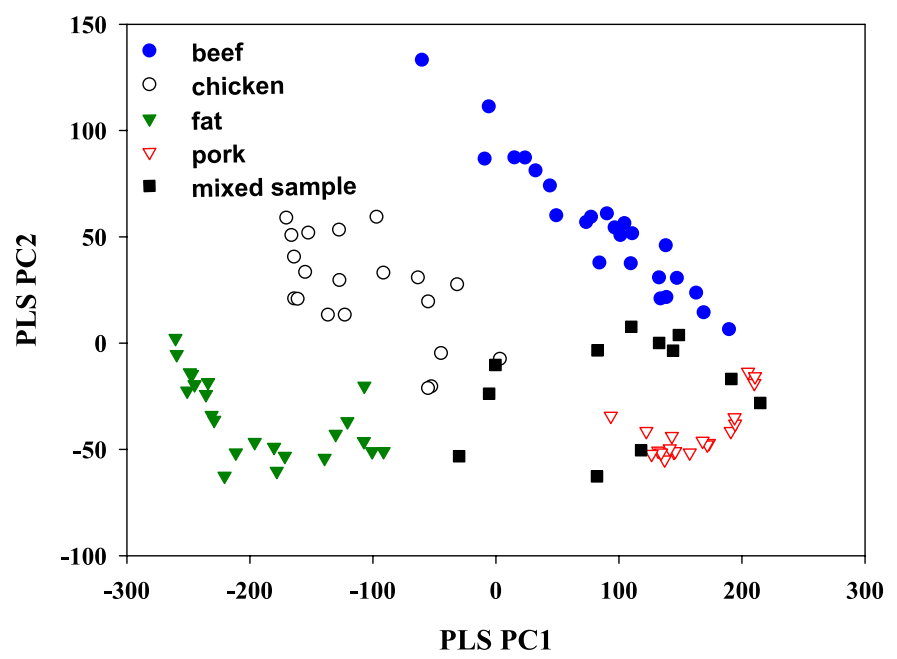

Fig. 7. Principal component plots using PLS analysis based on spectral analysis (414, 542, 655, $832 \mathrm{~nm})$. Each point indicates each unit. (Colors are visible in the online version of the article; http://dx.doi.org/10.3233/SPE-2011-0528.)

Table 2

Classification accuracy of the tissue type based on four discrete spectral bands $(414,542,655,832 \mathrm{~nm})$

\begin{tabular}{|c|c|c|c|c|c|}
\hline & & Corre & edicted class & & \\
\hline & Beef & Chicken & Fat & Pork & $\mathrm{B} / \mathrm{P}$ \\
\hline$\overline{\text { Beef }}$ & $\begin{array}{c}96.0 \\
(\mathrm{CL} 1=96.0, \\
\text { CL2 }=0.0, \\
\text { CL3 }=0.0)\end{array}$ & 0.0 & 0.0 & 0.0 & 4.0 \\
\hline Chicken & 0.0 & $\begin{array}{c}100.0 \\
(C L 1=80, \\
\text { CL2 }=15, \\
\text { CL3 }=5)\end{array}$ & 0.0 & 0.0 & 0.0 \\
\hline Fat & 0.0 & 0.0 & $\begin{array}{c}100.0 \\
(C L 1=85, \\
\text { CL2 }=15, \\
\text { CL3 }=0)\end{array}$ & 0.0 & 0.0 \\
\hline Pork & 0.0 & 0.0 & 0.0 & $\begin{array}{c}100.0 \\
(\mathrm{CL} 1=91.3, \\
\text { CL2 }=8.7, \\
\text { CL3 }=0)\end{array}$ & 0.0 \\
\hline
\end{tabular}

Notes: $\mathrm{B}$ - beef, $\mathrm{C}$ - chicken breast, $\mathrm{P}$ - pork, $\mathrm{F}$ - fat and B/P - beef and pork at the same time. $\mathrm{CL}$ certainty level, CL1 - satisfactory, CL2 - marginally satisfactory, CL3 - poorly satisfactory.

portions. Naturally they were classified as pork. P095 that had more fat than pork was regarded as fat. In general, although very evident, the mixed unit was classified by a tissue type of larger area. If we have a smaller unit size, we can have a higher spatial resolution. One can choose an optimal unit size of classification depending on actual target size and clinical environment. 


\section{Conclusions}

Several tissue types in the microscope image could be classified with a very high accuracy. Different shades of red meat such as beef and pork, bloodless chicken breast and fat were selected in making composite-tissue images. The method was based on a partial least squares regression modeling where a particular tissue type was assigned 1 (yes) or 0 (no) and regression was performed. Each classification area in the image had discrete spectral-band intensities. We ran a PLS2 model by which the tissue types (beef, pork, chicken breast, fat) were regressed. Classification accuracy was poor when we used RGB colors. Apparently substantial spectral overlapping among RGB colors was blamed for this result. When we used four discrete bandpass filters over an entire CMOS detector range whose pass bands did not interfere at all one another, the classification accuracy was higher than $96 \%$.

In principal, it is better to have a complete spectrum over a wide range at each pixel like in hyperspectral imaging. However, it is impractical to implement such a wide-band optical system in terms of size and cost. Computational burden may not be able to process data in real time in case of capsule endoscopy. The proposed algorithm based on discrete-band spectroscopy can be immediately applied to bleeding detection in capsule endoscopy. We believe that our study can be clinically useful for other applications as well if we are able to choose filters and the pixel size within clinically acceptable accuracies.

\section{Acknowledgement}

This work has been supported by Bilateral international cooperative research and development program, Ministry of Knowledge Economy, Republic of Korea.

\section{References}

[1] V.K. Asari, Microprocess. Microsyst. 26 (2002), 161-171.

[2] S.C. Chu, T.-C.R. Hsiao, J.K. Lin, C.-Y. Wang and H.K. Chiang, IEEE Trans. Biomed. Eng. 53 (2006), 2265-2273.

[3] Z. Du, M.K. Jeong and S.G. Kong, IEEE Trans. Automat. Sci. Eng. 4 (2007), 332-339.

[4] Y. Hamamoto, T. Endo, K. Nosho, Y. Arimura, M. Sato and K. Imai, J. Gastroenterol. 39 (2004), 14-20.

[5] M. Hirata, S. Tanaka, S. Oka, I. Kaneko, S. Yoshida, M. Yoshihara and K. Chayama, Gastrointestinal Endoscopy 66 (2007), 945-952.

[6] http://www.intromedic.com.

[7] M.L. Huebschman, R.A. Schultz and H.R. Garner, IEEE Eng. Med. Biol. 21 (2002), 104-117.

[8] J. Hutchings, C. Kendall, N. Stone, N. Sheperd and H. Barr, J. Biomed. Opt. 15 (2010), 066015.

[9] M.A. Kara, M. Ennahachi, P. Fockens, F.J.W. ten Kate and J.J.G.H.M. Bergman, Gastrointestinal Endoscopy 64 (2006), 155-166.

[10] H. Kim, I. Noh and G. Yoon, J. Optic. Soc. Korea 13 (2009), 279-285.

[11] W. Konen, S. Tombrock and M. Scholz, Med. Image Anal. 11 (2007), 526-539.

[12] S. Maitra and J. Yan, in: 2008 Casualty Actuarial Society: Applying Multivariate Statistical Model, Quebec City, Canada, June 15-18, 2008, pp. 79-90.

[13] D. Manolakis and G. Shaw, IEEE Signal Process. Mag. 19 (2002), 29-43.

[14] R. Miranda-Luna, C. Daul, W.C.P.M. Blondel, Y. Hernandez-Mier, D. Wolf and F. Guillemin, IEEE Trans. Biomed. Eng. 55 (2008), 541-553.

[15] G. Muller, W. Wasche, U. Bindig and K. Liebold, Laser Phys. 9 (1999), 348-356.

[16] E.J. Seibel, R.E. Carroll, J.A. Dominitz, R.S. Johnston, C.D. Melville, C.M. Lee, S.M. Seitz and M.B. Kimmey, IEEE Trans. Biomed. Eng. 55 (2008), 1032-1042.

[17] I.V. Skornyakov, G.B. Tolstorozhev and V.Z. Butra, J. Appl. Spectrosc. 75 (2008), 420-425.

[18] T. Smith and J. Guild, Trans. Opt. Soc. 33 (1931), 73-134.

[19] Y. Xian, K. Xu, Z. Zhang, Y. Dai and P.B. de Harrington, J. Biomed. Opt. 15 (2010), 067002.

[20] X. Yuan, N. Situ and G. Zouridakis, Pattern Recognition 42 (2009), 1017-1028. 


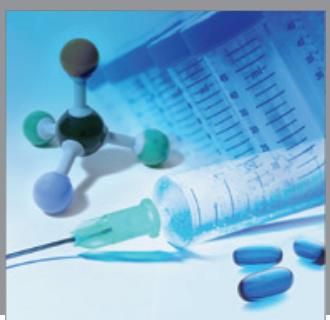

International Journal of

Medicinal Chemistry

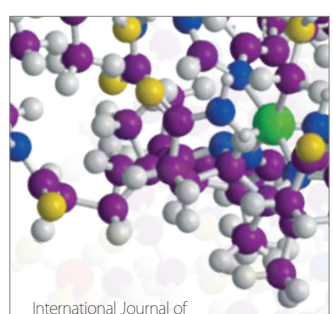

Carbohydrate Chemistry

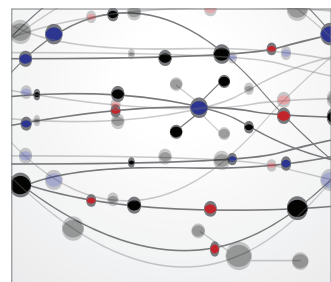

The Scientific World Journal
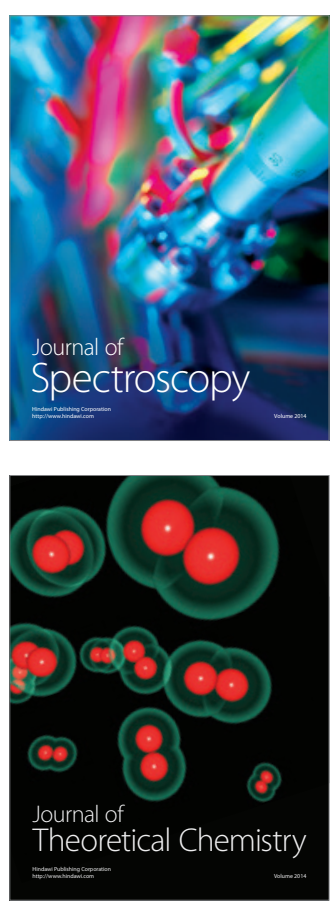
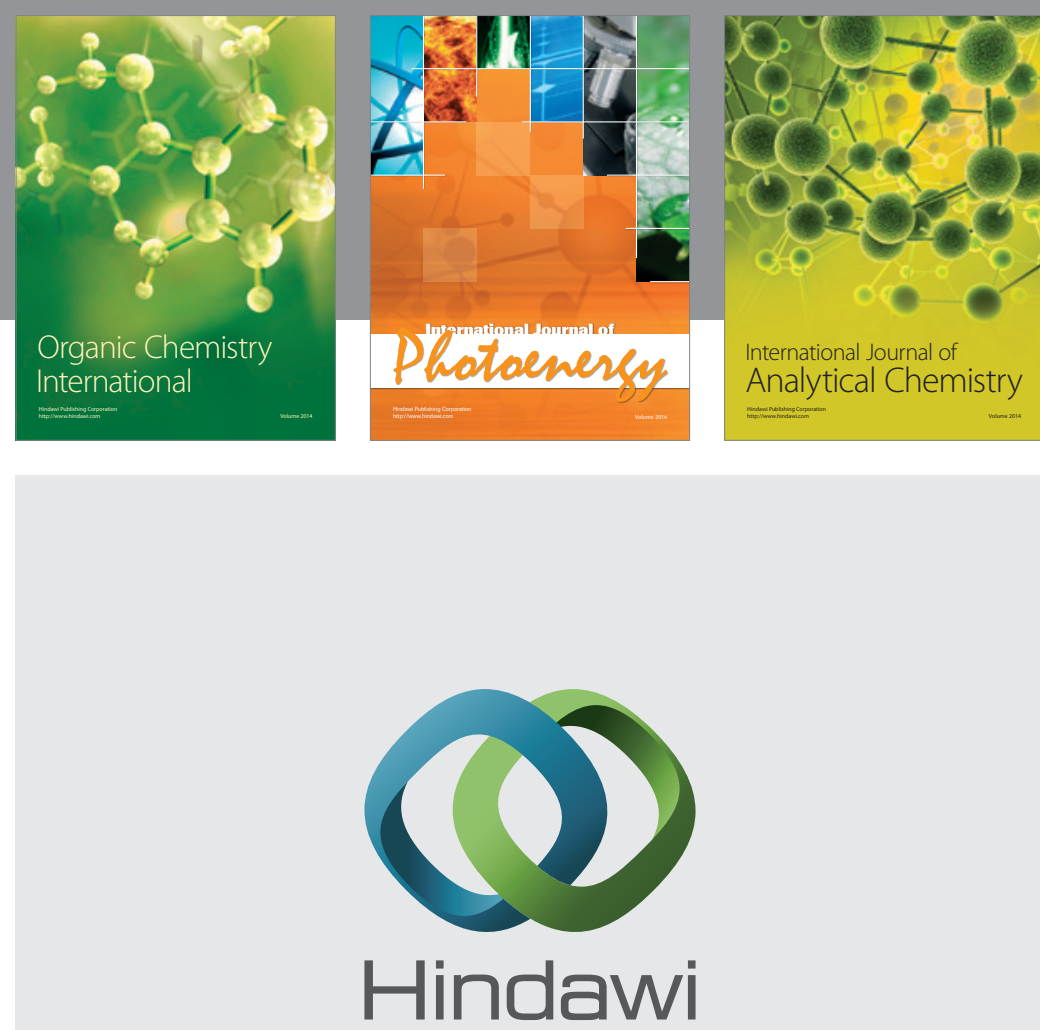

Submit your manuscripts at

http://www.hindawi.com
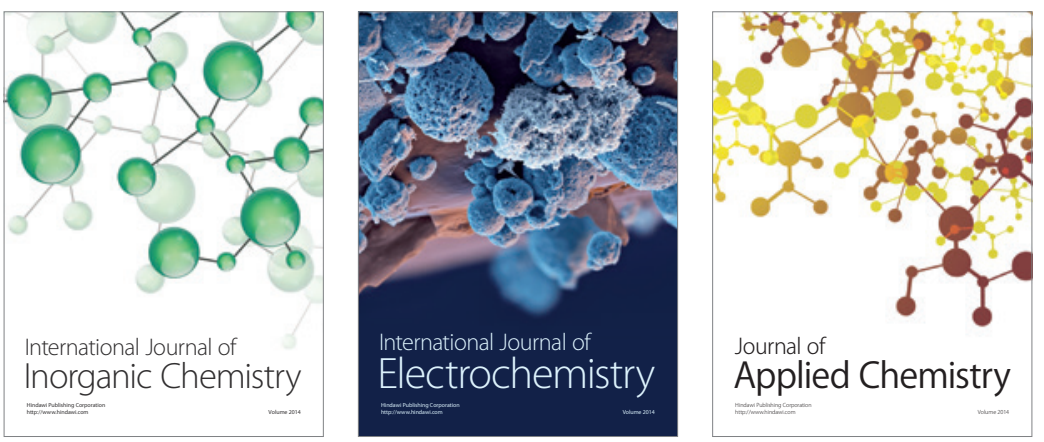

Journal of

Applied Chemistry
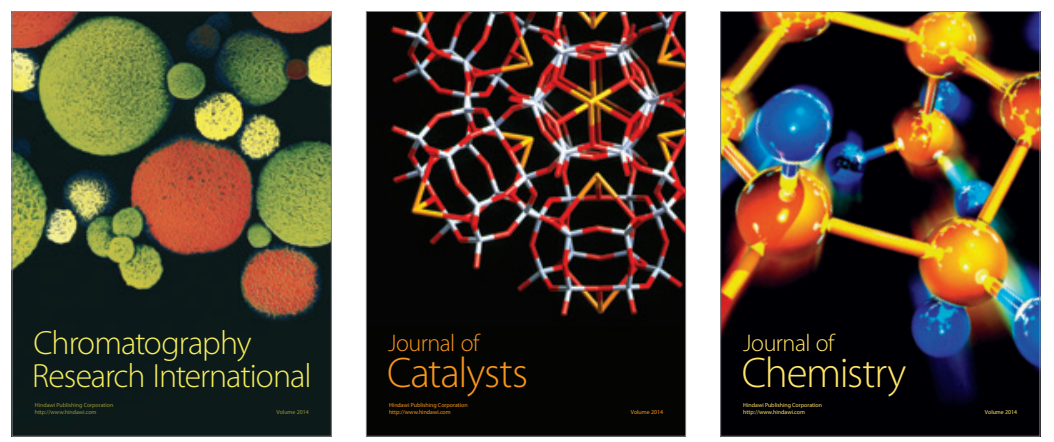
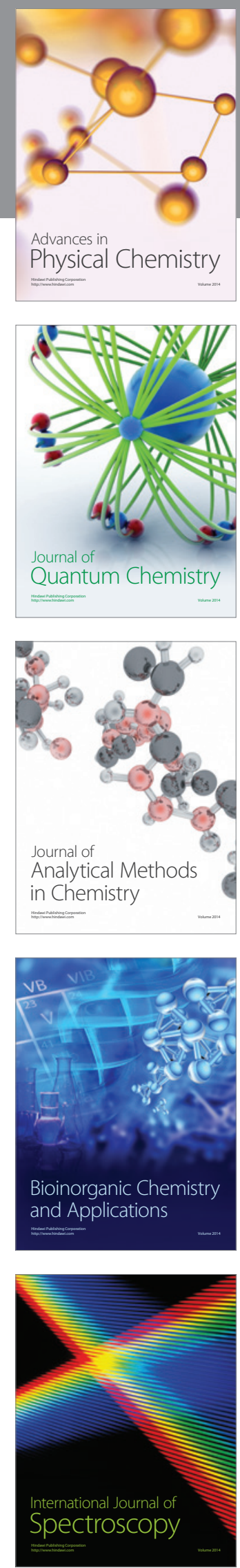